\title{
Social Risk Early Warning of Environmental Damage of Large-Scale Construction Projects in China Based on Network Governance and LSTM Model
}

\author{
Junmin Fang $\mathbb{D},^{1,2}$ Dechun Huang, ${ }^{1,2}$ and Jingrong $\mathrm{Xu} \mathbb{i D}^{1}$ \\ ${ }^{1}$ Business School, Hohai University, Nanjing 211100, China \\ ${ }^{2}$ Jiangsu Provincial Collaborative Innovation Center of World Water Valley and Water Ecological Civilization, \\ Nanjing 211100, China
}

Correspondence should be addressed to Jingrong Xu; xujingrong@hhu.edu.cn

Received 17 September 2020; Revised 10 November 2020; Accepted 15 November 2020; Published 26 November 2020

Academic Editor: Guangdong Wu

Copyright (C) 2020 Junmin Fang et al. This is an open access article distributed under the Creative Commons Attribution License, which permits unrestricted use, distribution, and reproduction in any medium, provided the original work is properly cited.

\begin{abstract}
With the improvement of citizens' risk perception ability and environmental protection awareness, social conflicts caused by environmental problems in large-scale construction projects are becoming more and more frequent. Traditional social risk prevention management has some defects in obtaining risk data, such as limited coverage, poor availability, and insufficient timeliness, which makes it impossible to realize effective early warning of social risks in the era of big data. This paper focuses on the three environments of diversification of stakeholders, risk media, and big data era. The evolution characteristics of the social risk of environmental damage of large-scale construction projects are analyzed from the four stages of incubation, outbreak, mitigation, and regression in essence. On this basis, a social risk early warning model is constructed, and the multicenter network governance mode of social risk of environmental damage in large-scale construction projects and practical social risk prevention strategies in different stages are put forward. Experiments show that the long short-term memory neural network model is effective and feasible for predicting the social risk trend of environmental damage of large-scale construction projects. Compared with other classical models, the long short-term memory model has the advantages of strong processing capability and high early warning accuracy for time-sensitive data and will have broad application prospects in the field of risk control research. By using the network governance framework and long short-term memory model, this paper studies the environmental mass events of large-scale construction projects on the risk early warning method, providing reference for the government to effectively prevent and control social risk of environmental damage of large-scale construction project in China.
\end{abstract}

\section{Introduction}

Large-scale construction projects play an important role in the economic, political, cultural, and ecological environment, with the characteristics of multiagent participation, long period of construction and operation, and many factors involved, such as water conservancy, industrial construction, and real estate projects. In the implementation process, large-scale construction project will involve issues related to ecological environment, land expropriation and demolition, migration, and interest groups; it may cause social conflicts and collective conflicts among stakeholders such as government, project contractor, and the public. With the gradual improvement of the public's education level, risk perception ability, and environmental protection awareness, environmental disputes have become a new factor that tends to intensify social conflicts and affect social harmony. In recent years, environmental pollution problems caused by large-scale construction project or social conflicts caused by environmentally sensitive projects have expanded at an average annual rate of $29 \%$ [1]. In order to seek economic benefits, many regions and enterprises have built environmental risk projects with higher economic profits. Risk perception and risk amplification are the main inducements leading to the outbreak of social risk events. The conflicts between stakeholders and social contradictions caused by 
them are significantly higher than those of other types of risk events in terms of confrontation degree. Therefore, it is particularly urgent to scientifically identify the social risk development stage of large-scale construction project environmental damage and systematically construct the social risk early warning mechanism.

Social conflicts caused by environmental damage generally have the characteristics of national conditions in China, and people's information sources are increasingly "controlled" by media. These mainstream media tools provide a speech path for citizens' environmental expression, such as WeChat, forums, and microblogs, to provide new communication channels for the social risks of environmental damage of the large-scale construction project. Meanwhile, the Internet has strong characteristics of openness, rapid communication, and interaction, which makes the risks that could have been controlled under traditional communication channels, to be amplified through media tools and gradually transformed into social risk events, triggering social public crises, and bringing great pressure and challenges to the government and other public management departments. At present, the related research on risk control of large-scale construction projects mainly focuses on the research on the relationship between interest subjects of large-scale construction projects [2], the understanding of the social risk spread and diffusion law of construction project environmental damage by traditional construction project impact, and social risk management theory. As the most critical step in the social risk early warning process of current large-scale construction projects, the relevant research and practice are far behind the current Web 2.0 (big data) environment of public crisis early warning reality needs [3]; no matter in terms of risk analysis methods or technical support, it cannot provide automatic, intelligent, and real-time crisis early warning support for the current dynamic and interactive social risks.

\section{Literature Review}

Construction project is a "double-edged sword" to boost economic and social development. With the enhancement of consciousness of sustainable development, it is impossible to avoid the negative impact of construction on ecological environment while promoting social and economic development [4]. Judging from the behavior causing environmental damage, the construction project will bring about pressure to the ecological environment system from two aspects: on the one hand, it is environmental pollution behavior; its common characteristic is that the material or energy discharged into the environment during the construction and operation stages of the construction project exceeds the self-purification ability of the environment, leading to the decline of environmental quality [5]. Previous studies have shown that traditional construction methods are the main causes of environmental pollution, and construction is the main source of carbon emissions [6, 7]. On the other hand, the ecological destruction behavior of construction projects refers to the exhaustion of resources and degradation of ecological functions caused by the speed and intensity of resource consumption exceeding the ecological carrying capacity [8]. The ecosystem has the function of "resilience"; when the external interference exceeds a certain threshold, the resilience function is lost and the ecosystem enters an unstable state. According to statistics, about $67.5 \%$ and $21 \%$ of Malaysia's ecosystems and natural resources are affected by construction activities of construction projects [9].

The results of environmental pollution and ecological destruction are superimposed together and cause and affect each other [10]. Judging from the objective damage results caused by the environmental damage behavior of large-scale construction projects, it can be summarized as the damage of public rights and environmental interests [11]. Research shows that the root cause of social conflicts caused by environmental changes is the imbalance of interests caused by environmental degradation [12]. The public within the scope of environmental pollution is the main stakeholder, not only direct stakeholders but also third-party social forces such as environmental protection organizations, public welfare personages, experts, and scholars [13]. They use the immeasurable damage caused by environmental deterioration to express their own interests and emotional catharsis to the local government. Once the contradiction intensifies due to improper handling, it is easy to produce agglomeration behavior and turn into more serious social harm [14]. The social conflicts caused by the interaction of environmental effects and social effects are becoming the "fuse" of social conflicts. The environmental risks of large-scale construction projects are likely to transform into social risks, and the degree of social risks depends on the response of the vulnerability and adaptability of social systems to environmental degradation [15].

"Large-scale construction projects cause environmental damage and then social instability" is a complicated process. Entering the modern media society, informatization and networking make people more and more accustomed to transmitting information and exchanging emotions in the virtual society. In particular, the emergence of new Internet media has significantly improved people's ability to feel, recognize, and respond to social risks [16]. Social amplification of risk refers to the social experience of how information process, institutional structure, social group behavior, and individual reaction shape risk, thus leading to risk consequences [17]. The China Environmental Development Report (2014) points out that the real risks of some environmental pollution projects protested by the public may not be as strong as those perceived by the public, and even some are low-risk projects, indicating that there is an obvious "risk amplification mechanism" in the field of environmental damage of large-scale construction project [18]. The Social Amplification of Risk Framework (SARF) provides a tool for studying the communication of social risks in media society, which can better understand how risk information is transmitted and changed through different actions and interactions [19]. However, SARF was formulated 30 years ago, far earlier than the emergence of the network media environment as is known it today. Therefore, it is studied in traditional mass media to a large extent. 
Recently, scholars have begun to consider the framework of online media and social media $[20,21]$.

Digital communications provide new opportunities for researchers to study concepts and frameworks such as SARF in ways that were previously impossible $[22,23]$. Online communication enables researchers to collect data and analyze how individuals and groups communicate on these platforms, including through comment areas on news websites and social media sites [24]. Due to the characteristics of large scale, large number of participants, rapid dissemination, and rich data content of social media data and the analysis and modeling of information dissemination in social media data have become a difficulty in research [25]. This paper focuses primarily on the transfer of information stage as a first step in rethinking the framework in the times of social media and Web 2.0 information environments. Within the transfer of information, there are several mechanisms that may contribute to social amplification of risk: the volume of media coverage, amount of attention by information consumers, how controversial or disputed the information is, how dramatized the information is, and the channels of information involved [26, 27]. The understanding of risks is not obtained through direct experience to a large extent. Risk disseminators, especially mass media, play an important role in the construction and interpretation of risk issues while disseminating risk information [28]. Existing literature shows that the media have a significant influence on public perception, attitude, and response to risks [29]. Extensive media coverage of controversial environmental issues has aroused social concern or public concern about risks, even if the media's position on these issues is balanced. In newspapers, action, television, and other broadcast media, real or dramatic descriptions of risks and disasters may affect viewers' perception and judgment of risks [30, 31].

Risk is a kind of loss caused by uncertainty; it can help stakeholders accurately identify risk changes, assess risks, and make timely risk response measures by establishing a risk early warning mechanism, which is in line with the original intention of establishing other similar warnings and has sufficient practical significance [32]. In general, risk early warning should have functional modules of risk information collection, identification and assessment, warning, and response and realize the functions of risk identification, early warning, response, and immune risk [33]. The development of information technology and big data has given rise to a new evolution path of risks. In recent years, many studies have combined different kinds of machine learning methods in different forms $[34,35]$. For example, neural network, Bayesian network, support vector machine, and other machine learning methods as the center of the framework, combined with other advanced technologies in the field of research, are very rich [36, 37]. For example, in order to overcome the shortcomings of traditional food risk early warning models in terms of hypothesis, sample size, and generalization ability, Zhang [38] proposed a hybrid early warning model based on Logit and SVM to effectively describe the linear and nonlinear features of customer default influenced by different factors. Takeda A [39] constructed a support vector machine-based and minimax probability machine-based classification and regression models to forewarn financial risks. Data and information are updated rapidly in the new era. Machine learning involves probability theory, statistics, approximation theory, convex analysis, algorithm complexity theory, and other interdisciplinary theories and is widely used in artificial intelligence, financial and economic data analysis, DNA biological genetic data analysis, and other fields [40-42]. The combination of qualitative research and quantitative analysis forms a set of theoretical research paradigm, which has the ability to solve practical problems to a certain extent. Good results were achieved in various fields [43]. The era of big data is the necessity of social development and has more and more influence on human activities. Big data early warning of individual and collective behaviors has become feasible and necessary. In the big data environment, social mass incidents are no longer prevented and controlled by the government as a single subject but are jointly managed by the government, society, market, and other multiple subjects [44]. At present, the application of social risk early warning is currently in the exploratory stage. Hence, how to quickly discover valuable data and master its regularities of distribution and risk prevention are the next research directions.

\section{Analysis on Social Risk Evolution of Environmental Damage in Large-Scale Construction Projects}

Timely collection and discovery of warning information before and after the occurrence of social risk events is the key to the early warning of social risks of environmental damage in large-scale construction projects. It can accurately predict and grasp the evolution of social risks and the crisis situation of specific events. As the information representation of social risk dissemination, network public opinion is an important source of risk early warning information. Relying on the full data characteristics of the Internet, it is meaningful to screen the information of mass incidents and accurately warn and control the trend. These data point to the trend of risk evolution.

\subsection{Social Risk Evolution Process of Environmental Damage in} Large-Scale Construction Projects. The social risk events of construction projects caused by environmental problems have their own laws of development and evolution, which can be distinguished by the stage division method of the life cycle. Like other things with life, it not only goes through the evolution process from beginning to end and from origin to disappearance but also is divided into several stages from its generation and development to decline, corresponding to different periods of the life cycle. Figure 1 shows changes in network attention of social risk events of large-scale construction projects caused by environmental problems; the social conflicts caused by environmental problems of these six projects have great social influence in China from 2012 to 2019. With the development of time and nodes in the case of critical events, the social risk transmission of environmental 


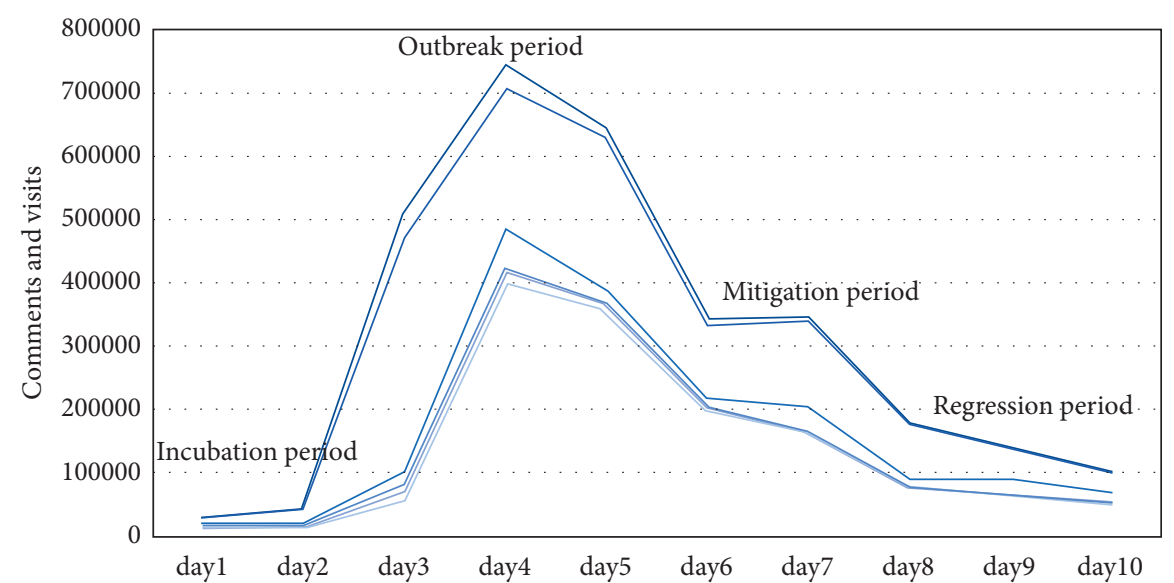

Figure 1: Changes of social attention to social conflict events of environmental damage in large-scale construction projects.

damage in large-scale construction projects presents a chainlike development and evolution process. This acceleration of this chain reaction gradually increases, allowing social risks that were not originally seen to exceed their safety thresholds to reach the ignition point and explode, which is a process of gradual formation-aggregation-outbreak $[45,46]$. The social risk evolution of environmental damage in different types of large-scale construction projects generally goes through four periods.

3.1.1. Incubation Period. Incubation period of social risks refers to the possibility that social risk sources appear and exist in society and lead to social losses only after certain conditions, which also refers to the period from the emergence to the evolution of risk sources. The greatest feature is that it is not confrontational or destructive. As the factors that cause social conflicts already exist, such as some social contradictions or social problems (corruption, demolition, gap between rich and poor, etc.) that are concerned by the society, social risk events have not really occurred, but there are some hidden risk signals that are difficult to find. At this time, social risks have not been transformed from "hidden" to "explicit."

3.1.2. Outbreak Period. At this stage, social conflicts and contradictions continue to increase, and social pressure cannot be effectively released, resulting in an increasing severity of risks. When all conditions are ripe, social crises will outbreak once encountering emergency. Obstruction of interest demands is a necessary condition to trigger such risk events. When the stakeholders of social conflict need to further express interest demands, the subjects expand the social influence of the conflict through the "acquaintances" such as relatives, friends, classmates, and colleagues. Attracting more subjects to participate, the outbreak of risks means that social risks have undergone qualitative changes. Mass events are the result of the continuous amplification and evolution of social risks of environmental damage in large-scale construction projects. At this time, the state of social risks has evolved to the state of social crisis.
3.1.3. Mitigation Period. Under the interference and influence of internal and external uncertainties, the deviation on a certain node of social risks has led to the amplification and enhancement of social risks and has been transmitted and spread to various points and surfaces in the formation process of social risks in various ways, causing social development to deviate from the track or cause chaos. During this period, the government has taken emergency postevent control measures to curb the spread of social risks. Through the control of events and the weakening of risk transmission, the social risk evolution of environmental damage in largescale construction projects will enter a mitigation period.

3.1.4. Regression Period. After the social risk of environmental damage of large-scale construction projects broke out, the driving force rapidly weakened until it died out. The high efficiency of social risk transmission in the big data environment determines that any single independent social risk conflict event will soon be obliterated by other more eyecatching events and disappear from the public screen. In addition, the rapid and accurate intervention and response of government departments will also quickly fade away the public opinion on the social risks of environmental damage of large-scale construction projects.

\subsection{Stakeholder Analysis of Social Risk of Environmental} Damage in Large-Scale Construction Projects. Analyzing and summarizing the research on the evolution of social risks by different scholars at domestic and foreign research status, it is not difficult to find that the starting point of social conflicts is the conflicts of interest between different stakeholders [47]. Due to the complexity of large-scale construction projects, there are many subjects involved. To study the social conflicts of environmental damage of large-scale construction projects, the first step is to define the multiple stakeholders, that is, the stakeholders of social risks of environmental damage of large-scale construction projects. Stanford Research Institute, Freeman [48], and many other institutions and scholars believe that stakeholders include not only those individuals and groups that directly affect the 
organization's goals but also government departments, social organizations, and the public that are related to the organization, because these groups will also have an impact on the process of achieving the organization's goals. Therefore, these related subjects that affect the process and goal realization of large-scale construction projects can also be called stakeholders of large-scale construction projects. In China, social risks are usually related to mass incidents, social conflicts, and violent resistance. In the process of planning and construction of large-scale construction projects, sometimes the interests of other subjects are ignored, resulting in conflicts of interest and social risks, which further affect the realization of project objectives and even affect social security and stability. Therefore, the relationship between stakeholders and social risks should be paid full attention.

There are many factors affecting the social risks of largescale construction projects, and the relationship with various stakeholders is also very close, forming a complex network relationship and affecting the formation and diffusion of social risks of large-scale construction projects. In China, large-scale construction projects have the characteristics of large influence on the public and long duration. The relations involved in land acquisition and relocation, resettlement, destruction of living environment, destruction of ethnic cultural traditions, and influence of vulnerable groups are very complicated. The interests and contradictions of different subjects are integrated, mainly including the conflicts between public officials seeking personal interests and public interests in engineering construction as well as the conflicts of interest demands of different subjects such as government, project legal persons, relocated households, and immigrants. The following are involved: contractors, local people, subcontractors, suppliers, social teams, environmental protection departments, supervision units, operators, design units, consumers, environmentalists, media, project peripheral organizations, owner units, banks, employees, immigrants, governments, consulting units, and other stakeholders. Some stakeholders appear in most construction projects, while others only appear in specific construction projects. The contradiction between these stakeholders has become more intense with the spread of the project construction cycle and scale. Once the interest demands of various subjects cannot be satisfied, social contradictions will be further intensified. However, in the process of social risk evolution caused by environmental damage of large-scale construction projects, due to the characteristics of such projects, the main investment and management subjects are generally the government, including the local government and the superior government. The builder is the construction enterprises, responsible for part of the project investment, specific construction affairs, and the completion and operation management, etc. Other stakeholders include the subjects that can affect the interests related to the construction of the project except the government and the project legal person. These subjects are the damaged subjects that are most likely to cause social risks due to the loss of interests caused by the project, mainly including the public in the project location and the public in the nonproject location. On the other hand, as the information transmission will be less and less restricted by time and space in the Internet era, the mass media and the interpersonal network among various subjects have increased the impact of psychological perception on the public, enhanced the diffusion and spillover of the fuse of stakeholder conflicts of social risks of environmental damage of largescale construction projects, more easily aroused social attention, and accelerated the amplification of social risks. In summary, in the process of social risks caused by environmental damage of large-scale construction projects, there are mainly four stakeholders: the government (local and superior), the construction enterprises, the public where the project is located, and the media. In order to maximize their respective interests, these key stakeholders participate in the mutual interest game. Therefore, based on the perspective of stakeholder theory, this paper combines the relevant literature and the previous research basis to select the government, construction enterprises, the public, and the media as the main stakeholders of social risks of environmental damage. From three aspects of risk amplification, social conflicts, and policy games, the evolution process of social risks is revealed in combination with the behaviors of interest subjects in different periods, as shown in Figure 2.

\section{Social Risk Early Warning Model for Environmental Damage of Large-Scale Construction Projects}

4.1. Construction of the Social Risk Early Warning Indicator System. On the basis of scientifically grasping the laws and characteristics of media communication of the generation, development, and change of social risks of environmental damage in large-scale construction projects, the use of big data technology can realize the comprehensive collection and monitoring of information of various factors such as stakeholders, regions involved, demands and wishes, target orientation, conflict nature, influencing factors, emotional changes, onlookers, development trends, and correlation degree. According to the existing data types, the corresponding data indicators are designed to build a social risk early warning model.

In the process of constructing the social risk early warning indicator system for large-scale construction projects, the least number of indicators can achieve the greatest early warning benefit as much as possible. This section constructs the three first-level indicators and 11 second-level indicators in the process of literature review, which are shown in detail in Table 1 . The primary indicator corresponds to the three dimensions of the social risk event diffusion of the environmental damage of the project under the new media environment: the social risk perception of environmental damage, the performance of risk information, and the state of risk diffusion. During the time from the emergence of conflict signs to the beginning of risk events and causing perceptible losses, the law of risk communication brewing, development, outbreak, change, and dissipation is grasped. The secondary indicator is the 


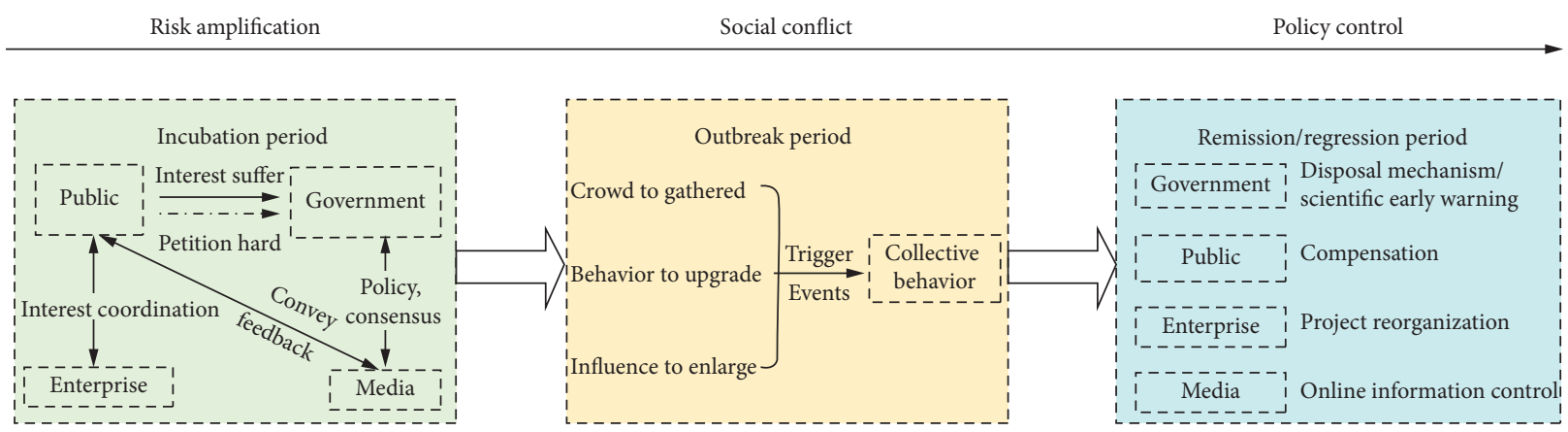

FIGURE 2: Behavior analysis of stakeholders in different stages of social risk evolution of environmental damage of large-scale construction project.

TABle 1: Social risk early warning index system for environmental damage of large-scale construction projects.

\begin{tabular}{|c|c|c|}
\hline Primary indicator & Secondary indicators & Definitions \\
\hline \multirow{4}{*}{ Risk perception } & Online search & $\begin{array}{l}\text { Baidu Index reflects the exposure and user attention of different keywords in the past } \\
\text { period and describes social hotspots, interests, and needs of all circles. }\end{array}$ \\
\hline & Original Weibo & $\begin{array}{c}\text { Original Sina Weibo related to environmental damage of large-scale construction } \\
\text { project. }\end{array}$ \\
\hline & Forwarding & $\begin{array}{c}\text { The number of reposts on Weibo indicates that they agree with the opinions of original } \\
\text { users. }\end{array}$ \\
\hline & Comment & $\begin{array}{c}\text { The number of comments on Weibo indicates that they agree with the opinions of } \\
\text { original users. }\end{array}$ \\
\hline \multirow{3}{*}{$\begin{array}{l}\text { Performance of risk } \\
\text { information }\end{array}$} & Point of view & $\begin{array}{l}\text { Refers to the user's attitude towards the environmental damage of the project, such as } \\
\text { approval, neutrality, and opposition. This indicator is mainly expressed by the ratio of } \\
\text { negative expression to all Weibo. }\end{array}$ \\
\hline & $\begin{array}{l}\text { Information } \\
\text { authenticity }\end{array}$ & $\begin{array}{c}\text { The percentage of users who certify users through Sina. In generally, the authenticated } \\
\text { user is responsible for the authenticity of his speech and will not distribute false } \\
\text { information easily. }\end{array}$ \\
\hline & Risk transmission & $\begin{array}{l}\text { Refers to the user's willingness to share information with other users. The higher the } \\
\text { frequency of @ other people, the wider the scope of information dissemination. }\end{array}$ \\
\hline \multirow{4}{*}{ Risk diffusion state } & $\begin{array}{l}\text { The gradient of online } \\
\text { search }\end{array}$ & $(Q 2-Q 1) /(T 1-T 2)$. The online search amount at time $T 1$ is $Q 1$, and $T 2$ is $Q 2$. \\
\hline & $\begin{array}{l}\text { The gradient of original } \\
\text { Weibo }\end{array}$ & $(Q 2-Q 1) /(T 1-T 2)$. The original Weibo amount at time $T 1$ is $Q 1$, and $T 2$ is $Q 2$. \\
\hline & $\begin{array}{l}\text { The gradient of } \\
\text { forwarding }\end{array}$ & $(Q 2-Q 1) /(T 1-T 2)$. The forwarding amount at time $T 1$ is $Q 1$, and $T 2$ is $Q 2$. \\
\hline & $\begin{array}{l}\text { The gradient of } \\
\text { comment }\end{array}$ & $(Q 2-Q 1) /(T 1-T 2)$. The comment amount at time $T 1$ is $Q 1$, and $T 2$ is $Q 2$. \\
\hline
\end{tabular}

decomposition of the internal structure of the primary indicator, to ensure that it can be quantified and be suitable for computer automation $[49,50]$. The data comes from Baidu Index and Sina Weibo, the world's largest Chinese search engine and social network platform, respectively.

\subsection{Analysis of Long Short-Time Memory Risk Early Warning} Model. The time sequences prediction method based on machine learning has strong self-learning and self-adaptive ability and shows stronger prediction ability when dealing with linear and nonlinear sequence modeling. For the traditional neural network, there is no time dimension connection between the input data, but this connection is crucial for the problems of risk signal prediction, commodity sales status, article understanding, and machine translation. Different from the fully connected neural network or convolution neural network model, the chain structure of
Recurrent Neural Network (RNN) has strong signal feedback capability to transfer information from one neuron of the network to the next neuron of the network through the hidden layer. However, the general RNN has the problem of gradient disappearance. In order to solve this problem, the long short-term memory (LSTM) retains the external chain structure of RNN and adjusts the internal structure. The overall structure of LSTM is very similar to RNN, and there is a chain structure. However, the repeated modules in LSTM are different from RNN. Compared with the traditional RNN network, LSTM network has a very useful mechanism, namely, forgetting gate. Instead of using a single network layer in LSTM, four network layers are combined in a special way. The specific structure is shown in Figure 3. Different from the single tanh loop structure, LSTM uses three different thresholds (input gate, output gate, and forget gate) to control, which greatly enhances the long-term memory ability of the network and effectively solves the 


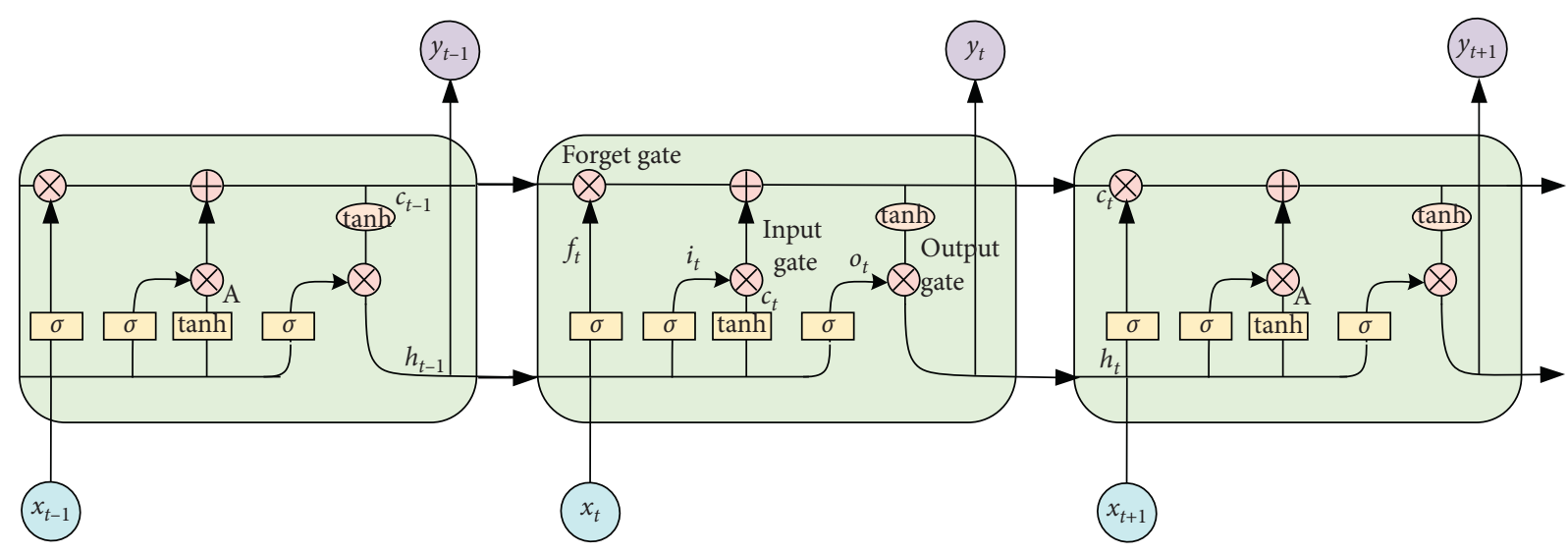

Figure 3: The sketch of the hidden layer of LSTM network.

problem of gradient disappearance. Meanwhile, the three thresholds of LSTM improve the ability of long-term information memory, filtering out relatively unimportant information to reduce training time. Because it can maintain long-term dependence in data, LSTM is widely used in practical applications. LSTM applications include text generation, machine translation, speech recognition, generation of image descriptions and video markers, and big data feature extraction.

The evolution process of social risk of environmental damage can be viewed as a time sequence. Therefore, RNN can be used to capture the important propagation characteristics of risk information, which judges them different from stage. However, RNN has the problems of gradient explosion and gradient vanishing, as well as position bias, which always tend to retain the information near the final location and weaken the earlier information. In order to avoid these problems, this paper chooses a special type of RNN, LSTM [51], to construct the risk early warning model and has intensity processing capability for time sequences data with relatively long intervals and delays. Figure 3 shows the structure of the hidden layer of LSTM, and the core is processing the cell state. The cell state runs through different time series operations. The state information is easily transmitted, and the information is added or removed through some linear interactions. The linear interaction is mainly realized by the gate structure; after multiplication by the Sigmoid neural network layer and the element level, the results are judged to achieve the transfer control of the cell state. The input value of LSTM network is the index system constructed in this paper, which has 11 inputs, and the output value is the four stages of social risk evolution, which has 4 outputs, as the LSTM basic steps presented as follows:

(1) The LSTM determines which information is output from the previous state and deletes the unimportant information through the Sigmoid activation function of the forget gate. The input of the forget gate includes the previous state $h_{t-1}$ and the current input $x_{t}$ (referring to the $t$ element in the sequence), and the value is output through the activation function, ranging from 0 to 1 . Finally, the result is multiplied by the previous cell state $C_{t-1}$ and output.

(2) Adding new useful information to the cell state through the input gate and then inputting $h_{t-1}$ and the current state input $x_{t}$ into the sigmoid function to delete the unimportant information, while $h_{t-1}$ and $x_{t}$ get a range between -1 and 1 through the tanh function, it generates a new candidate value in this step and determines whether to add it to the cell state subsequently.

(3) By multiplying the output of the sigmoid function and the tanh function in the second step and adding the output result in the first step, it is possible to achieve important information that is retained. Currently, update the status $C_{t}$ to forget the unimportant information.

(4) The previous hidden state $h_{t-1}$ and the current input $x_{t}$ go through the Sigmoid function to get the result $O_{t}$, which ranges from 0 to 1 . The output value of the tanh function is calculated with the output result of the third step, multiplied by $O_{t}$ as the output result $h_{t}$ of the current cell state and the input value of the next hidden state $h_{t-1}$. Finally, important information is selected from the current state as an output of the cell state. The specific mathematical formulae are as shown in the six following equations:

the Sigmoid activation function:

$$
\sigma(x)=\frac{1}{1+e^{-x}},
$$

input gate: $\mathbf{i}_{t}=\sigma\left(w_{i} \times x_{t}+R_{i} \times h_{t-1}+E_{i} \times c_{t-1}+b_{i}\right)$,

forget gate: $f_{t}=\sigma\left(w_{f} \times x_{t}+R_{f} \times h_{t-1}+E_{f} \times c_{t-1}+b_{f}\right)$,

output gate: $o_{t}=\sigma\left(w_{y} \times x_{t}+R_{y} \times h_{t-1}+E_{y} \times c_{t-1}+b_{y}\right)$, 


$$
\text { cell state: } \begin{aligned}
c_{t} & =f_{t} \times c_{t-1}+i_{t} \times \overline{c_{t}}, \\
\overline{c_{t}} & =\sigma\left(w_{c} \times x_{t}+R_{c} \times h_{t-1}+b_{c}\right),
\end{aligned}
$$

output vector: $h_{t}=o_{t} \times \sigma\left(c_{t}\right)$.

4.3. Parameter Setting. This paper uses the TensorFlow platform to implement the model building and training process, and LSTMCell operation is provided in TensorFlow to support the LSTM model building. LSTMCell is equivalent to the hidden layer of LSTM model. It encapsulates the forgetting gate, input gate, output gate, and other structures contained in the hidden layer of LSTM. At the same time, it can also set the number of hidden layer nodes according to research needs. In the process of building the neural network with TensorFlow, the layout is no longer based on the nodes in the neural network but on the layer. In particular, the purpose of dropout operation of input data is to prevent the model from being too fitted. The main training parameters in the model include the weight and skew from the input layer to the hidden layer, the weight and skew of the three gates in LSTMCell, and the weight and skew of the logistic regression layer. After the loss function cost is given, using the training operation provided by TensorFlow, the differential derivative of cost with respect to each parameter can be automatically obtained and the model can be trained by gradient descent method. The output layer nodes are set to 4 , corresponding to the 4-degree levels of social risk early warning. The settings for the other parameters are given in Table 2. The initialization of training parameters in the model will have a great influence on the training effect, and the random-uniform-initializer is provided by TensorFlow to initialize the training parameters of the logistic regression layer. The random-uniform-initialize method is used to initialize the parameters of forgetting gate, input gate, and output gate in LSTMCell. In addition, when creating a new LSTMCell, adjusting the parameter "forget bias" from the default 0 to 1.0 will improve the training effect of the model. This paper uses Batch Gradient Descent method for training.

\subsection{Case Study}

4.4.1. Data Source. Combined with the four-stage model of social risk of environmental damage in large-scale construction projects, the early warning level is divided into four levels according to the National General Emergency Plan for Public Emergencies, level I (especially serious), level II (serious), level III (heavy), and level IV (general), to use 1000, 0100, 0010, and 0001 to output four alert level states, respectively. In this paper, the social risk level of construction project environmental damage is related to the stage of risk information dissemination, and the social risk incubation period of construction project environmental damage is defined as the heavier stage, the outbreak stage is defined as the especially serious stage, the mitigation stage is defined as the serious stage, and the regression stage is defined as the general stage. According to the established social risk early
TABle 2: Parameter setting.

\begin{tabular}{lc}
\hline Parameter & Value \\
\hline Number of nodes in input layer & 11 \\
Input & 4 \\
Sequence length & 256 \\
Number of hidden layers & 1 \\
Number of hidden layer nodes in each layer & 128 \\
Learning rate & 0.001 \\
Number of instances per training & 10 \\
Forget bias & 1.0 \\
\hline
\end{tabular}

warning index system, the social conflict events caused by environmental problems of influential large-scale construction projects in China from 2012 to 2019 were selected. The cases selected in the paper all have the following common characteristics: (1) caused by environmental damage caused by the construction of large construction projects; (2) a certain number and scale; (3) involves many departments and industries with multiple subject; (4) fierce expression and internal contradictions confrontation; and (5) high social concern. These social risk mass events of environmental damage of large-scale construction projects are typical representatives with great influence and high social concern in China within ten years. These mass events all have the same characteristics: sensitivity, news, and influence, so these 6 typical cases are selected for analysis. The data are of typical cases such as Qidong event in Jiangsu province, Yuhang, Zhongtai waste incineration event, and petrochemical project in Maoming, Guangdong province. According to the law of social risk transmission of environmental damage, the sample is divided into two parts, which are used for training and testing, respectively. Because the dimensions of the last-level indexes are different, in order to facilitate processing and comparison, the original data are normalized $[0,1]$ (4 decimal places are reserved).

4.4.2. Accuracy of Calculation. The samples in different stages of mass events caused by social risks of environmental damage of each large-scale construction project were sorted according to category labels during the experiment. Then put the samples from different stages of the event into the test level, and select the other $30 \%$ of the samples not to be labeled into the training set. We set up to iterate the training model 10,000 times with all actions of the training set, and each iteration will randomly rearrange the training set data. In this way, the loss function value of the model after each iteration can be recorded, and the recorded result is shown in Figure 4. It can be seen from the figure that the loss function value decreases with iteration and finally tends to be stable, which is also in line with the general training process of the neural network model. In the process of 10,000 iterations, the accuracy rate of the model on test data is as high as $90 \%$.

4.4.3. Simulation Results. Based on the construction principle of LSTM neural network, the input value of LSTM network is the index system constructed in this paper, which 


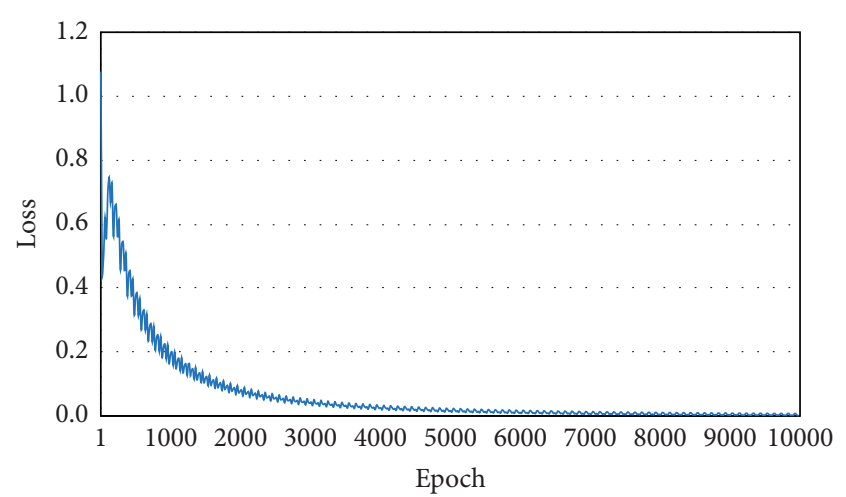

FIgure 4: The curve of the loss function with the number of iterations.

has 11 inputs, and the output value is the four stages of social risk evolution, which has 4 outputs. The conversion results of actual output of training data are compared with the expected output values to observe their consistency, which indicates that the model is effective; otherwise, the construction of the neural network is unreasonable and needs further improvement. The experimental results show that the actual output conversion results are basically consistent with the expected output. Different warning signals are issued at different stages of social risks, including blue warning signal (1000) in incubation period, red warning signal (0100) in outbreak period, orange warning signal (0010) in mitigation period, and green warning signal (0001) in regression period. The recording results are shown in Figure 5. The predicted output is a columnar bar, and the actual value is an orange polyline. The development trend of mass incidents of environmental damage in construction projects can be clearly observed from the figure. The prediction output of the model basically conforms to the actual early warning state and conforms to the evolution law of social risks. The experimental results demonstrate that the social risk early warning index system for environmental damage of large-scale construction projects constructed in this paper is basically reasonable, and the social risk early warning model based on LSTM neural network is effective. It can accurately warn the social risk evolution stage of environmental damage of large-scale construction projects and provide important basis for the government to take risk control decisions.

\section{Network Governance Strategy of Social Risk of Environmental Damage of Large-Scale Construction Projects}

Social risk governance is gradually showing a highly complicated, dynamic, and uncertain development trend. As a new social risk governance model, network governance has obvious advantages different from the traditional social risk governance model. The core idea of network governance theory is that it is impossible for any country or social actor to unilaterally decide the public policy process and governance process. Social governance can only be realized

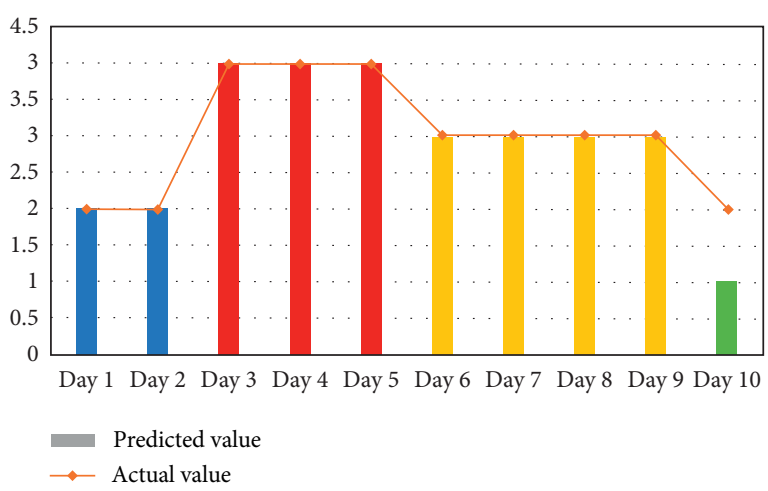

FIgURE 5: The simulation results of social risk early warning of environmental damage of large-scale construction project.

through close cooperation and joint participation of these actors [52]. Under the social background of risk media, the traditional social management mode with the government as the main body of management is a nonnegotiation and topdown management mode backed by the state's coercive force. It blocks the interaction and game, compromise, and coordination between the public and the government and ignores the fact that social governance needs to activate the combination of various stakeholders in the governance network. Therefore, in addition to clarifying the roles and responsibilities of stakeholders at social risks of environmental damage of large-scale construction projects, it is also important to clarify their mutual relations. This paper constructs a "multicenter network governance" mode involving multiple subjects such as government, enterprises, media, the public, and nongovernmental organizations, as shown in Figure 6. The social risk governance of environmental damage of construction projects is regarded as a multicenter network governance in which stakeholders cooperate with each other. On the one hand, the local government should organize horizontal linkage among different departments such as environmental protection, construction and information, and vertical linkage among different levels to efficiently respond internally. On the other hand, the government's external impact includes the realtime monitoring and evaluation of enterprises (previous studies often ignore the control of enterprises), as well as the disclosure of media information and the rapid monitoring of public attitudes. The mass events caused by environment are a dynamic process from social risk to public crisis. In order to deal with the social risk of environmental damage of largescale construction projects, in addition to properly handling the social crisis through appropriate strategies, it is also necessary to construct long-term governance strategies for social risks. Emphasize the interaction and communication between the government and the public and other stakeholders to realize the balance of rights and obligations among stakeholders.

On this basis, a social risk resolution mechanism for environmental damage of large-scale construction projects based on the integration of government and media and the guidance of public opinion is constructed. Through early warning of different development stages of social risks of 


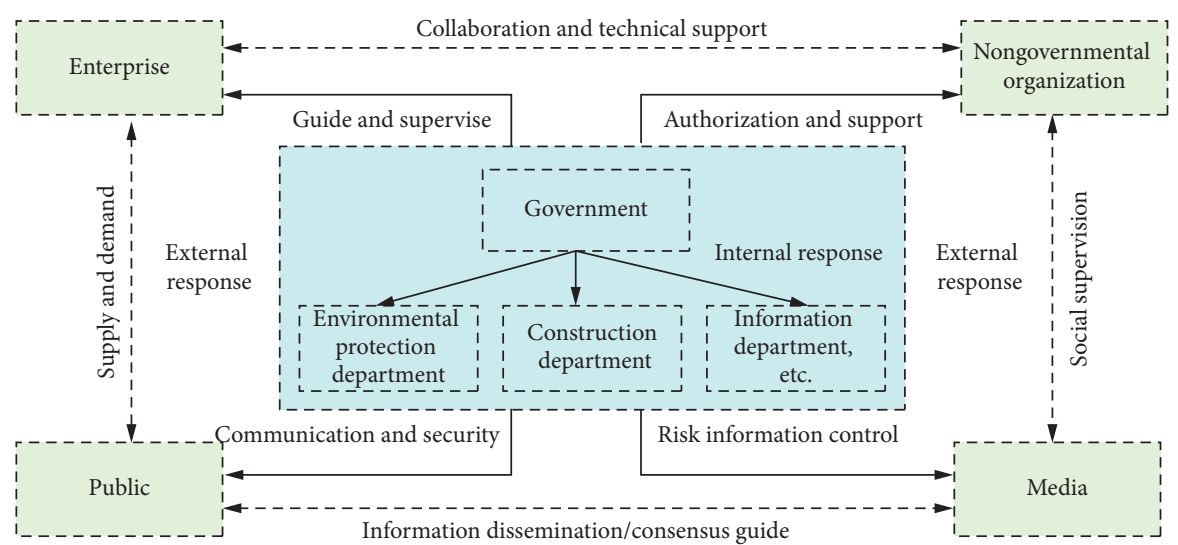

FIGURE 6: The multicenter network governance mode of social risks early warning of environmental damage of large-scale construction project.

environmental damage of large-scale construction projects, social risk resolution mechanisms for incubation period, outbreak period, mitigation period, and regression period are proposed, respectively:

(1) In incubation period, the government can grasp the opportunity of public opinion by timely disclosing information on environmental damage and social conflicts of large-scale construction projects. To guide the integration of network media and government media, on the one hand, the credibility of government media will be brought into play, relevant information will be released in a timely and dynamic manner, and relevant issues of social concern will be responded to. On the other hand, give full play to the advantages of network media, guide them to communicate with the public with a fair and true attitude, and resolve social conflicts caused by environmental damage of large-scale construction projects.

(2) In outbreak period, the government should properly handle the stakeholders in the mass events, for example, train "leaders" of the media to correctly guide the direction of public opinion. In the process of social risk diffusion, the comments made by individuals who are at the core of the social network and have more social relations have a greater impact on the public and are very easy to guide people's judgment. The government needs to attach importance to the role of leaders in the process of public opinion dissemination and obtain their support. Cultivate "opinion leaders" under the integration of the government and the media, so that opinion leaders who are more easily recognized by the public can communicate with the affected people, transmit correct ideas, channel the affected people to express their interest demands in a legal way, and ease social conflicts caused by environmental damage of largescale construction projects.

(3) In mitigation period, the government should take allround intervention measures, mobilize all forces to strengthen the release and dissemination of effective information, and make full use of the power of the public opinion field to dilute the concentration of risk information. When the public's interest demands are fermented simultaneously in the public opinion field of the government and the public, the two interact and jointly affect the social risk amplification of environmental damage of large-scale construction projects. Therefore, the power of the public opinion field should be used to change the unidirectionality of public opinion guidance to promote the effective communication and exchange of the subjects in the social conflict's events of environmental damage of large-scale construction projects. Through the media platform, the public can release their opinions and thoughts on social risk events, not only meeting the public's right to know but also enabling the government and enterprises to timely understand the public's interest demands at different stages, thus taking relevant measures in a timely manner and scientifically guiding public opinion.

(4) In regression period, the government should pay attention to the promotion of institutionalization. With the evolution of social risks, public opinions will gradually decline, but risks do not mean complete disappearance. At this stage, the influence of social risks began to dissipate, but the governance of social risk events did not end. If we do not strengthen the top-level design and improve the social risk early 
warning system for the mass events of environmental damage in this large-scale construction project, the influence and opinions lurking in the public mind will sprout again under the stimulus of new conflicts. As the main body of social risk early warning for environmental damage of large-scale construction projects, government departments should integrate relevant resources in social risk early warning, make overall plans to solve the outstanding problems existing in current management departments, and improve the social risk early warning system.

\section{Conclusions}

This paper refines the evolution stage from environmental damage of social risk of large-scale construction projects from the perspectives of diversification of interest subjects, risk media, and environment in the era of big data and analyzes the division of responsibilities and coordination mechanism of key stakeholders such as government, enterprises, media, and the public in different periods and constructs a social risk early warning model for environmental damage of large-scale construction projects, which provides a new perspective for social risk governance. The conclusions are listed as follows:

(1) In the process of social risk media, some new features have emerged in the dissemination of social conflict behaviors. For example, the interaction between individual stakeholders can change the form and path of social risk amplification, and network media can reshape the speed and stage of risk information dissemination. By analyzing the social risk mass events of construction projects caused by environmental problems in the past ten years, it is proved that the media communication of social risks is an objective existence and has its own law of development and evolution, which can be distinguished by the method of dividing stages of life cycle; the evolution of social risks will go through four stages: incubation, outbreak, mitigation, and regression. Combining the influence of stakeholders and risk media on risk evolution, the early warning timing of social risks of environmental damage in large-scale construction projects can be accurately divided, providing theoretical basis for the construction of early warning model. On the other hand, according to the different roles and responsibilities of the social risk management subjects for environmental damage of large-scale projects, the association between various stakeholders in the governance network is activated, so that they can participate in all stages of social risk management.

(2) Compared with general collective behavior, social risk events have short gestation period, concentrated outbreak time, many risk subjects, and short life cycle; the social risk data of environmental damage of construction projects have certain fragmentation and discontinuity characteristics. Combined with the phased propagation law and characteristics of social risk of environmental damage of large-scale construction projects, the evolution process of social risk is regarded as a time series. LSTM social risk early warning model can accurately capture the important propagation characteristics of risk information, effectively process time series data with long interval and large delay, and accurately realize early warning results by automatically learning the historical and current data of risk propagation. The source control, dynamic control, and whole-process control of risks are combined to transform postevent intervention into prewarning and take effective preventive actions, thus effectively preventing social risks of largescale construction environmental damage from management systems and technical means and finally realizing the overall goal of the social risk control system.

There are two limitations in this paper. Firstly, it only combines the social risk data of environmental damage of large-scale construction projects in China, which has certain limitations: in particular, some stakeholders deleted the published data after the social risk mass events subside. Secondly, the governance strategies of the four risk evolution stages proposed in this paper do not carry out policy simulation analysis and cannot evaluate their practicability and effectiveness. Further research in this paper should focus on the two following aspects: Firstly, further expand the scope of investigation, study more relevant cases at domestic and foreign levels, and use preprocessing of risk information, such as web page purification, text segmentation, and other means to improve the accuracy of information acquisition; moreover, the LSTM risk early warning model is improved by extending the data in the training process, such as adding noise data. Secondly, the simulation analysis of the risk management strategies should be carried out. We should suppose that the appropriate risk management strategies have been taken and increase the collection of relevant data and social investigation and then carry out characteristic analysis and effectiveness quantification of risk management strategies based on different stages to provide support for the optimization of risk management strategies.

\section{Data Availability}

The data used to support the finding of this study are included within the supplementary information files.

\section{Conflicts of Interest}

The authors declare no conflicts of interest.

\section{Acknowledgments}

This work was supported by the Postgraduate Research \& Practice Innovation Program of Jiangsu Province (no. KYCX19_0373), the Fundamental Research Funds for the Central Universities (no. 2019B68514), the National Natural Science Foundation of China (nos. 71573072 and 71603070), 
and Jiangsu Province Social Science Application Research Project (no. 20SYA-040).

\section{Supplementary Materials}

supplementary materials are submitted to the system, involving all the data of the case study of the manuscript in Section 4.4. In order to ensure the authenticity and validity of all data, the authors have provided the index data of all cases and explained each supplementary materials file. The authors can be contacted if there are any format requirements. Baidu Index: the social risk early warning indicators for environmental damage of large-scale construction projects. Baidu Index is one of the social risk early warning indicators for environmental damage of large-scale construction projects constructed in this paper. The data explain Baidu search volume of different nodes in a period of time for mass incidents of environmental damage caused by large-scale construction projects. The data comes from Baidu Index web page. Cases 1-6: In the case study of the manuscript in Section 4.4, the social conflict events caused by environmental problems of influential large-scale construction projects in China from 2012 to 2019 were selected: Qidong event in Jiangsu province, Yuhang, Zhongtai waste incineration event, petrochemical project in Maoming Guangdong province, Hongda molybdenum copper project in Shifang, Sichuan province, large-scale nuclear fuel processing plant in Jiangmen, Guangdong province, and PX project in Kunming province. According to the social risk early warning index system of environmental damage of large-scale construction projects constructed in this paper, the secondary index data come from Sina Weibo, respectively. The data of each case shows the data of relevant users of the mass incidents of environmental damage caused by large-scale construction project on Sina Weibo within a certain period of time by using big data mining tools. It ensures the authenticity and validity of each piece of data and provides the basis for the case study in Section 4.4. Preprocessed sample data: according to the social risk early warning model of environmental damage of large-scale construction projects constructed in this paper, training data and prediction data are needed in LSTM network. The case sample is divided into two parts, which are used for training and testing, respectively. Because the dimensions of the lastlevel indexes are different, in order to facilitate processing and comparison, the original data are normalized $[0,1](4$ decimal places are reserved). The preprocessed sample data is used as training data and test data of the LSTM model. (Supplementary Materials)

\section{References}

[1] S. S. Li, "Explanation of the Decision (Draft) of the Standing Committee of the National People's Congress on amending individual provisions of seven laws, including the Prison LawAt the 29th Session of the Standing Committee of the Eleventh National People's Congress on October 23, 2012," Bulletin of the Standing Committee of the National People's Congress of the People's Republic of China, vol. 2012, no. 6, pp. 720-721, 2012.
[2] Z. He, B. Wang, D. Huang et al., "Stakeholder conflict amplification of large-scale engineering projects in China: an evolutionary game model on complex networks," Complexity, vol. 2020, Article ID 9243427, 17 pages, 2020.

[3] L. Zhang, L. Xu, and W. Zhang, "Social media as amplification station: factors that influence the speed of online public response to health emergencies," Asian Journal of Communication, vol. 27, no. 3, pp. 322-338, 2017.

[4] Z. He, D. Huang, C. Zhang et al., "Toward a stakeholder perspective on social stability risk of large hydraulic engineering projects in China: a social network analysis," Sustainability, vol. 10, no. 4, pp. 12-23, 2018.

[5] S. Malik, F. Fatima, A. Imran et al., "Improved project control for sustainable development of construction sector to reduce environment risks," Journal of Cleaner Production, vol. 2019, no. 240, pp. 118-214, 2019.

[6] Y. Zhong and P. Wu, "Economic sustainability, environmental sustainability and constructability indicators related to concrete- and steel-projects," Journal of Cleaner Production, vol. 2015, no. 108, pp. 748-756, 2015.

[7] C. C. W. Hung, S.-C. Hsu, and K.-L. Cheng, "Quantifying city-scale carbon emissions of the construction sector based on multi-regional input-output analysis," Resources, Conservation and Recycling, vol. 2019, no. 149, pp. 75-85, 2019.

[8] R. Damania, J. Russ, D. Wheeler, and A. F. Barra, "The Road to Growth: Measuring the Tradeoffs between Economic Growth and Ecological Destruction," World Development Oxford, vol. 101, no. 1, pp. 748-756, 2018.

[9] R. Zakaria, K. A. Mohamed, R. M. Zin, and S. Zolfagharian, "Sustainable development factors for land development in Universiti Teknologi Malaysia's Campus," OIDA International Journal of Sustainable Development, vol. 3, no. 9, pp. 105-110, 2012.

[10] S. Chen, "Environmental pollution emissions, regional productivity growth and ecological economic development in China," China Economic Review, vol. 2015, no. 35, pp. 171-182, 2015.

[11] H. Campbell and A. Scott, "Costs of learning about the environmental damage of mining projects," Economic Record, vol. 2010, no. 56, pp. 134-152, 2010.

[12] G. Bennet, "Dilemmas: coping with environmental problems," Golden Research Thoughts, vol. 66, no. 15, pp. 164-165, 1992.

[13] R. G. Khlebopros, V. A. Okhonin, and A. I. Fet, "Environmental Damage, Catastrophes in Nature and Society," in Mathematical Modeling of Complex SystemsWorld Scientific Publishing (UK) Ltd, London, UK, 2014.

[14] H. E. Daly and C. W. Cobb, For the Common Good: Redirecting the Economy Toward Community, the Environment and a Sustainable Future, Vol. 2, Boston Massachusetts Beacon Press, Boston, MA, USA, 2017.

[15] A. R. Binder, D. A. Scheufele, D. Brossard et al., "Interpersonal amplification of risk? Citizen discussions and their impact on perceptions of risks and benefits of a biological research facility," Risk Analysis An Official Publication of the Society for Risk Analysis, vol. 31, no. 2, pp. 324-331, 2011.

[16] A. R. Binder, M. A. Cacciatore, D. A. Scheufele et al., "Measuring risk/benefit perceptions of emerging technologies and their potential impact on communication of public opinion toward science," Public Underst, vol. 21, no. 7, pp. 830-847, 2012.

[17] O. Renn, W. J. Burns, J. X. Kasperson et al., "The social amplification of risk: theoretical foundations and empirical 
applications," Journal of Social Issues, vol. 48, no. 4, pp. 137-160, 2010.

[18] M. A. Cacciatore, D. A. Scheufele, and S. Iyengar, "The end of framing as we know it and the future of media effects," Mass Communication \& Society, vol. 19, no. 1, pp. 7-23, 2016.

[19] R. E. Kasperson, O. Renn, P. Slovic et al., "The social amplification of risk: a conceptual framework," Risk Analysis, vol. 8, no. 2, pp. 177-187, 1988.

[20] J. Fellenor, J. Barnett, C. Potter et al., "The social amplification of risk on Twitter: the case of ash dieback disease in the United Kingdom," Journal of Risk Research, vol. 21, no. 10, pp. 1-21, 2018.

[21] Y. A. Strekalova and J. L. Krieger, "Beyond words: amplification of cancer risk communication on social media," Journal of Health Communication, vol. 22, no. 10, pp. 849857, 2017.

[22] B. Miles and S. Morse, "The role of news media in natural disaster risk and recovery," Ecological Economics, vol. 2017, no. 63, pp. 365-373, 2007.

[23] P. Piedrahita, J. Borge-Holthoefer, Y. Moreno et al., "The contagion effects of repeated activation in social networks," Social Networks, vol. 3, no. 11, pp. 1-12, 2017.

[24] D. L. Hansen, B. Shneiderman, M. A. Smith et al., "Social media: new technologies of collaboration," Analyzing Social Media Networks with NodeXL, Morgan Kaufmann, vol. 2020pp. 11-29, Burlington, MA, USA, 2nd edition, 2020.

[25] M. Imran, C. Castillo, F. Diaz, and S. Vieweg, "Processing social media messages in mass emergency: survey summary," Companion of the The Web Conference, vol. 2018, no. 4, pp. 507-511, 2018.

[26] J. M. Hansen, G. Saridakis, and V. Benson, "Risk, trust, and the interaction of perceived ease of use and behavioral control in predicting consumers' use of social media for transactions," Computers in Human Behavior, vol. 80, no. 3, pp. 197-206, 2018.

[27] C. Reuter, Marc-André Kaufhold, S. Schmid et al., "The impact of risk cultures: citizens' perception of social media use in emergencies across Europe," Technological Forecasting and Social Change, vol. 2019, no. 148, pp. 1-15, 2019.

[28] C. D. WirzM. A. Xenos et al., "Rethinking social amplification of risk: social media and zika in three languages," Risk Analysis, vol. 38, no. 12, pp. 2599-2624, 2018.

[29] I. J. Chung, "Social amplification of risk in the Internet environment," Risk Analysis, vol. 31, no. 12, pp. 1883-1896, 2011.

[30] Y. Guo and Y. Li, "Online amplification of air pollution risk perception: the moderating role of affect in information," Information, Communication \& Society, vol. 21, no. 1, pp. 80-93, 2018.

[31] M. Ivan, S. Ivana, and O. Nevenka, "Development of ANN model for wind speed prediction as a support for early warning system," Complexity, vol. 2017, Article ID 3418145, 10 pages, 2017.

[32] C. S. Jarnevich, T. R. Holcombe, D. T. Barnett et al., "Forecasting weed distributions using climate data: a GIS early warning tool," Invasive Plant Ence \& Management, vol. 3, no. 4, pp. 365-375, 2017.

[33] A. Bihorac, T. Ozrazgat-Baslanti, A. Ebadi et al., "MySurgeryRisk: development and validation of a machine-learning risk algorithm for major complications and death after surgery," Annals of Surgery, vol. 269, no. 4, pp. 652-662, 2018.

[34] W. Xu, Y. Chen, C. Coleman, and T. F. Coleman, "Moment matching machine learning methods for risk management of large variable annuity portfolios," Journal of Economic Dynamics and Control, vol. 87, no. 2, pp. 1-20, 2018.

[35] J. Li and L. Xu, "Comparison and analysis of research trend prediction models based on machine learning algorithm-BP neural network, support vector machine and LSTM model," Journal of Modern Information, vol. 39, no. 4, pp. 23-33, 2019.

[36] C. Xia and W. Yuan, "Research status and prospect of food safety risk early warning based on machine learning," Journal of Medical Informatics, vol. 40, no. 3, pp. 56-61, 2019.

[37] S. Yeom, I. Giacomelli, M. Fredrikson, and S. Jha, "Privacy Risk in Machine Learning: Analyzing the Connection to Overfitting," in Proceedings of the 2018 IEEE 31st Computer Security Foundations Symposium (CSF), pp. 268-282, Oxford, UK, July 2018.

[38] Q. Zhang, L. Y. Hu, and J. Wang, "Study on credit risk early warning based on Logit and SVM," System Engineering Theory and Practice, vol. 35, no. 7, pp. 1784-1790, 2015.

[39] A. Takeda and T. Kanamori, "Using financial risk measures for analyzing generalization performance of machine learning models," Neural Networks, vol. 57, no. 2, pp. 29-38, 2014.

[40] W. L. Bennett and S. Iyengar, "A new era of minimal effects? The changing foundations of political communication," Journal of Communication, vol. 58, no. 4, pp. 707-731, 2008.

[41] J. Kruppa, A. Ziegler, and I. R. KNig, "Risk estimation and risk prediction using machine-learning methods," Human Genetics, vol. 131, no. 10, pp. 1639-1654, 2012.

[42] B. Jonathan and C. Christopher, "Large sample size, wide variant spectrum, and advanced machine-learning Technique boost risk prediction for inflammatory bowel disease," American Journal of Human Genetics, vol. 92, no. 6, pp. 1008-1012, 2012.

[43] H. Cao, T. Lin, Y. Li, and H. Zhang, "Stock price pattern prediction based on complex network and machine learning," Complexity, vol. 2019, Article ID 4132485, 12 pages, 2019.

[44] G. U. Shen and S.-J. Wang, "Research of carbon financial risk early warning model based on SVM," East China Economic Management, vol. 2019, no. 3, pp. 1-15, 2019.

[45] W. Q. Wang, "Risk amplification, collective action and policy game: A descriptive analysis about environmental groups struggle violence," Journal of Public Management, vol. 12, no. 1, pp. 127-135, 2015.

[46] W. P. Yu, X. Zu, and Y. J. Meng, "Social risk inducement and management mechanism for major projects' environmental pollution: in the perspective of life cycle," Jilin University Journal Social Sciences Edition,vol. 56, no. 4, pp. 38-46, 2016.

[47] G. D. Oppong, A. P. Chan, and A. Dansoh, "A review of stakeholder management performance attributes in construction projects," International Journal of Project Management, vol. 20, no. 3, pp. 1037-1051, 2017.

[48] R. E. Freeman, "Strategic management: a stakholder approach," Journal of Management Studies, vol. 29, no. 2, pp. 131-154, 1984.

[49] Z. Liu, Z. Zhu, H. Wang, and J. Huang, "Handling social risks in government-driven mega project: an empirical case study from west China," International Journal of Project Management, vol. 34, no. 2, pp. 202-218, 2016.

[50] C. Wenpu, Z. Peng, W. Wei et al., "Research on the risk prevention and control mechanism of public opinion online related to fire departments," China Emergency Rescue, vol. 80, no. 2, pp. 37-41, 2020.

[51] F. Karim, S. Majumdar, H. Darabi, and S. Chen, "LSTM fully convolutional networks for time series classification," IEEE Access, vol. 6, no. 99, pp. 1662-1669, 2018.

[52] O. Berthod, M. Grothehammer, M. S. Gordon et al., "From high-reliability organizations to high-reliability networks: the dynamics of network governance in the face of emergency," Journal of Public Administration Research and Theory, vol. 2, no. 27, pp. 1-52, 2017. 